\title{
ON THE PRODUCT OF A CONTRACTIBLE TOPOLOGICAL MANIFOLD AND A CELL
}

\author{
BY MORRIS W. HIRSCH
}

Communicated by Edwin Spanier, June 22, 1962

1. Introduction. Our object is to prove the following theorem:

THEOREM. Let $M$ be a contractible manifold of dimension $m$.

(A) If $M$ has empty boundary, then $M \times R^{p} \approx R^{m+p}$ for some $p$.

(B) If $M$ is compact, then $M \times D^{q} \approx D^{m+q}$ for some $q$.

For a bound on $p$ and $q$, see $\S 4$.

Here $D^{i}$ denotes the closed unit disk in Euclidean $i$-space $R^{i}$, and $X \approx Y$ means $X$ is homeomorphic to $Y$. Our manifolds are not assumed to have any differential or combinatorial structures unless this is explicitly indicated. Throughout the paper $M$ denotes a manifold of dimension $m, \partial M$ is the boundary of $M$, and int $M=M-\partial M$.

2. Proof of (A). The analog of (A) for combinatorial manifolds is due to McMillan [4]. In view of this, to prove (A) it suffices to establish that $M \times R^{i}$ is homeomorphic to an open subset of $R^{m+j}$ for some $j$. The following result of Curtis and Lashof [3] is used for this purpose.

(C) Let $U \subset M \times M$ be a neighborhood of the diagonal. Let $\phi: U \rightarrow R^{m}$ be a map such that for all $y \in M, \phi(y, y)=0$ and $\phi \mid U_{y}$ is one-one, where $U_{y}=U \cap(M \times y)$. Then $M \times R^{i}$ can be embedded in $R^{m+i}$ for some $j$.

In the terminology of Milnor's theory of microbundles, the existence of $\phi$ is equivalent to the triviality of the tangent microbundle of $M$. In his paper [5], Milnor deals with combinatorial microbundles, but as he points out, much of the development applies to the topological case. In particular, it is true that homotopic maps induce equivalent microbundles. Therefore the tangent microbundle of a contractible manifold is trivial. Thus (C) may be used to complete the proof of $(\mathrm{A})$.

3. Proof of (B). If $M$ is contractible so is int $M$. Thus (A) applies to int $M$, and to prove (B) it suffices to demonstrate the following.

(D) Let $M$ be contractible. If (int $M) \times R^{k} \approx R^{m+k}$, then $M \times D^{k+1}$ $\approx D^{m+k+1}$.

The proof depends on some work of M. Brown.

(E) For any $M$, there is a neighborhood of $\partial M$ in $M$ homeomorphic to $(\partial M) \times D^{1}$.

This is proved in [2].

Put $S^{n-1}=\partial D^{n}=$ unit sphere in $R^{n}$. 
(F) Let $f: S^{n-1} \rightarrow R^{n}$ be an embedding. If there is a neighborhood $N$ of $S^{n-1}$ in $R^{n}$ and an extension of $g$ to an embedding of $N$, then $g$ can be extended to an embedding of $D^{n}$.

This is proved in [1] and in [6].

(G) Let $M$ be a compact manifold covered by two open sets $A_{1}$ and $A_{2}$, with $A_{1} \approx A_{2} \approx R^{m}$. Then $M \approx S^{m}$.

This is due to Stallings [10], and follows easily from (F).

Next we deduce some simple consequences of these theorems. First, for a manifold $M$, define the double of $M$ to be $\partial\left(M \times D^{1}\right)$, and denote it by $2 M$. It is clear that $2 M$ is homeomorphic to two copies of $M$ identified along $\partial M$.

(H) Let $M$ be a compact manifold with int $M \approx R^{m}$. Then $2 M \approx S^{m}$.

Proof. This is easily proved using (E) and (G).

(I) Suppose int $M \approx R^{m}$ and $\partial M \approx S^{m-1}$. Then $M \approx D^{m}$.

Proof. Follows easily from (E) and (F).

Proof of (D). Assume now that $M$ is compact and contractible, and that (int $M$ ) $\times R^{k} \approx R^{m+k}$. Then we have

(J) int $\left(M \times D^{k}\right) \approx R^{m+k}$;

(K) int $\left(M \times D^{k+1}\right) \approx R^{m+k+1}$.

From $(\mathrm{J})$ and $(\mathrm{H})$ we get

(L) $\partial\left(M \times D^{k} \times D^{1}\right) \approx S^{m+k+1}$.

Now by combining (I), (K), and (L), we see that $M \times D^{k+1} \approx D^{m+k+1}$, which proves (D).

4. A bound for $p$ and $q$. A bound for the dimensions $p$ and $q$ appearing in (A) and (B) can be found as follows. The $j$ in (C) is equal to the dimension of a Euclidean space in which $M$ is embedded as a neighborhood retract. Thus in (C), $j \leqq 2 m+1$. We can avoid a further Euclidean factor by applying results due to Stallings [9] in place of McMillan's theorem; these results imply that $M \times R^{i} \approx R^{m+i}$. Therefore:

(M) In (A) we may take $p \leqq 2 m+1$. In (B) we may take $q \leqq 2 m+2$.

5. Analogs. The differential analog of (A) follows from the combinatorial one [4] and uniqueness of compatible differential structures on contractible combinatorial manifolds, proved by Munkres [7]. The combinatorial and differential analogs of (B) are easy consequences of difficult theorems of Smale [8].

\section{REFERENCES}

1. M. Brown, A proof of the generalized Schoenflies theorem, Bull. Amer. Math. Soc. 66 (1960), 74-76.

2. - Locally flat embeddings of topological manifolds, Topology of 3-manifolds and related topics, Prentice Hall, Englewood Cliffs, N. J., 1962, pp. 83-91. 
3. M. Curtis and R. Lashof, On product and bundle neighborhoods, Proc. Amer. Math. Soc. (to appear).

4. D. McMillan, Cartesian products of contractible open manifolds, Bull. Amer. Math. Soc. 67 (1961), 510-514.

5. J. Milnor, Microbundles and differentiable structures, Princeton University, 1961 (mimeographed).

6. M. Morse, A reduction of the Schoenflies extension problem, Bull. Amer. Math. Soc. 66 (1960), 113-115.

7. J. Munkres, Obstructions to the smoothing of piecewise-differentiable homeomorphisms, Ann. of Math. 72 (1960), 521-554.

8. S. Smale, Differentiable and combinatorial structures on manifolds, Ann. of Math. 74 (1961), 498-502.

9. J. Stallings, The piecewise-linear structure of Euclidean space, Institute for Advanced Study and Princeton University, 1962 (mimeographed).

10. — - Polyhedral homotopy spheres, Bull. Amer. Math. Soc. 66 (1960), $485-488$.

University of California, Berkeley 